Bull. Mater. Sci., Vol. 22, No. 5, August 1999, pp. 835-842. (C) Indian Academy of Sciences.

\title{
Crystal growth study using combination of molecular dynamics and Monte Carlo methods
}

\author{
MASAO DOYAMA \\ Department of Materials, Teikyo University of Science and Technology, Uenohara, Yamanashi 409-0193, Japan
}

\begin{abstract}
Molecular dynamics method although provides details of energies of the system as a function of time, is not suited to simulate the processes involving activation processes. Therefore, we attempted to combine the molecular dynamics and Monte Carlo methods. Using molecular dynamics, the energies of the system were calculated which were subsequently combined with Monte Carlo method using random numbers, epitaxial growth of (111) plane of copper, silver, and gold. While surface adsorption and surface diffusion for copper, silver, and gold were simulated by use of molecular dynamics method, the relation between the growth rate of thin films and the packing density of atoms were obtained using Monte Carlo simulation. Thus, by combining the results of the molecular dynamics method and the Monte Carlo method the growth process of thin films at elevated temperatures were obtained, which is too tedious to be calculated by molecular dynamics alone.
\end{abstract}

Keywords. Homoepitaxial growth; thin films; embedded-atom method.

\section{Introduction}

Crystal growth is becoming increasingly important in diverse industries, particularly in the electronics industries. Molecular dynamics method is often used to simulate elemental process of surface diffusion (Takano $e t$ al 1998). In molecular dynamics studies, the energies of the system, including surface, is calculated as a function of time. The temperature of a specimen with the average kinetic energy is adjusted to an aiming temperature. For the surface diffusion simulation, a diffusing atom is held at a position, the stress is released (the positions of atoms are moved toward resultant force) and the energy of the system is calculated. However, the time step in the molecular dynamics normally used is of the order of $10^{-15} \mathrm{sec}$. Therefore, even in the case wherein one million time steps were calculated, the total elapsed time was only of the order of $10^{-9} \mathrm{sec}$. Normally surface diffusion occurs by the activation process, and therefore $10^{-9} \mathrm{sec}$ is not long enough to simulate many activation processes. In an activated process, usually many attempts are required before the saddle point of the activated process can be overcome. The molecular dynamics method, involving activation process, requires a long time of computations, and hence here is an attempt to combine the molecular dynamics and Monte Carlo methods. Using molecular dynamics, the energies of the system are calculated and subsequently combined with Monte Carlo method using random numbers, epitaxial growth of (111) plane of copper, silver, and gold.

Choice of the jumping sites for diffusing atoms is given by Monte Carlo method. In this study the energies of surface diffusion are calculated by molecular method and from this calculation the frequency of the diffusing atom is calculated from the curvature near the stable point. The next jumping position is selected by using random numbers.

\section{The potential used}

In metals, the conducting electrons travel from one atom to another atom and the interaction between atoms cannot be represented by a pairwise potential but by many body potentials. The interaction between the ith atom and the jth atom depends not only on the distance between them but also on other factors. By the embedded function (Foiles 1986; Ackland et al 1987; Oh and Johnson 1988, 1989; Baskes 1992), surface problems can also be treated. By the N-body embedded function used in Baskes (1992), the total energy is given by

$$
\begin{aligned}
& E_{\text {total }}=\Sigma E_{\mathrm{i}}, \\
& r_{\mathrm{ij}}=\left[r_{\mathrm{i}}-r_{\mathrm{j}}\right], \\
& E_{\mathrm{i}}=(1 / 2) \Sigma \phi\left(r_{\mathrm{ij}}\right)+F\left(\rho_{\mathrm{i}}\right), \\
& F\left(\rho_{\mathrm{i}}\right)=D \rho_{\mathrm{i}} \operatorname{In} \rho_{\mathrm{i}}, \\
& \rho_{\mathrm{i}}=\Sigma f\left(r_{\mathrm{ij}}\right) .
\end{aligned}
$$

Here, $E_{\text {total }}$ is the total internal energy of a crystal. $E_{\mathrm{i}}$ the internal energy associated with atom $i . \rho_{i}$ is the electron density at atom i due to all the other atoms. $F\left(\rho_{i}\right)$ is the embedding energy of an atom into electron density $\rho_{\mathrm{i}}$. $\phi\left(r_{\mathrm{ij}}\right)$ is the two-body central potential between atoms $\mathrm{i}$ and $\mathrm{j}$ separated by $r_{\mathrm{ij}}$ and is repulsive if $\left(r_{\mathrm{ij}}\right)$ is the 
contribution to the electron density at atom $\mathbf{i}$ due to atom $\mathbf{j}$ at the distance $r_{\mathrm{ij}}$ from atom i. $F\left(\rho_{\mathrm{i}}\right)$ is an attractive term. For the functional forms

$$
\begin{aligned}
& \phi\left(r_{\mathrm{ij}}\right)=A_{1}\left(r_{\mathrm{c} 1}-r_{\mathrm{ij}}\right)^{2} \exp \left(-C_{1} r_{\mathrm{ij}}\right), \\
& f\left(r_{\mathrm{ij}}\right)=A_{2}\left(r_{\mathrm{c} 2}-r_{\mathrm{ij}}\right)^{2} \exp \left(-C_{2} r_{\mathrm{ij}}\right),
\end{aligned}
$$

are assumed. $\phi\left(r_{\mathrm{ij}}\right)$ and $f\left(r_{\mathrm{ij}}\right)$ are smoothly truncated at $r_{\mathrm{c} 1}$ and $r_{\mathrm{c} 2}$, respectively. $r_{\mathrm{c} 1}$ was chosen to be $1.65 d$ ( $d$ is the nearest neighbour distance), $f\left(r_{i j}\right)$ was cut off between the next nearest neighbour and the third nearest neighbour for a perfect crystal. $r_{\mathrm{c} 2}$ was chosen to be $1.95 d$, i.e. $\phi\left(r_{\mathrm{ij}}\right)$ was cut off between the third nearest and the fourth nearest neighbours. The parameters of the potential are given in table 1 .

\section{Molecular dynamics}

The following results have been obtained using molecular dynamics. Figure 1 shows some of the motion and conversion of adatoms and adatom clusters.

Table 1. Potential parameters.

\begin{tabular}{lccc}
\hline & $\mathrm{Cu}$ & $\mathrm{Ag}$ & $\mathrm{Au}$ \\
\hline $\mathrm{A}_{1}$ & $8.28945997705 \times 10^{3}$ & $9.10810768184 \times 10^{3}$ & $5.18021878920 \times 10^{5}$ \\
$\mathrm{~A}_{2}$ & $1.83251035107 \times 10^{-2}$ & $5.25514178418 \times 10^{-2}$ & $1.83065797788 \times 10^{-2}$ \\
$\mathrm{C}_{1}$ & 10.72729128641 & 10.817215101079 & 15.57704835828 \\
$\mathrm{C}_{2}$ & $3.19759369823 \times 10^{-1}$ & 1.38555356572 & $1.28671308081 \times 10^{-5}$ \\
$\mathrm{D}$ & 13.07921251628 & 11.28152486706 & 11.93177438785 \\
\hline
\end{tabular}

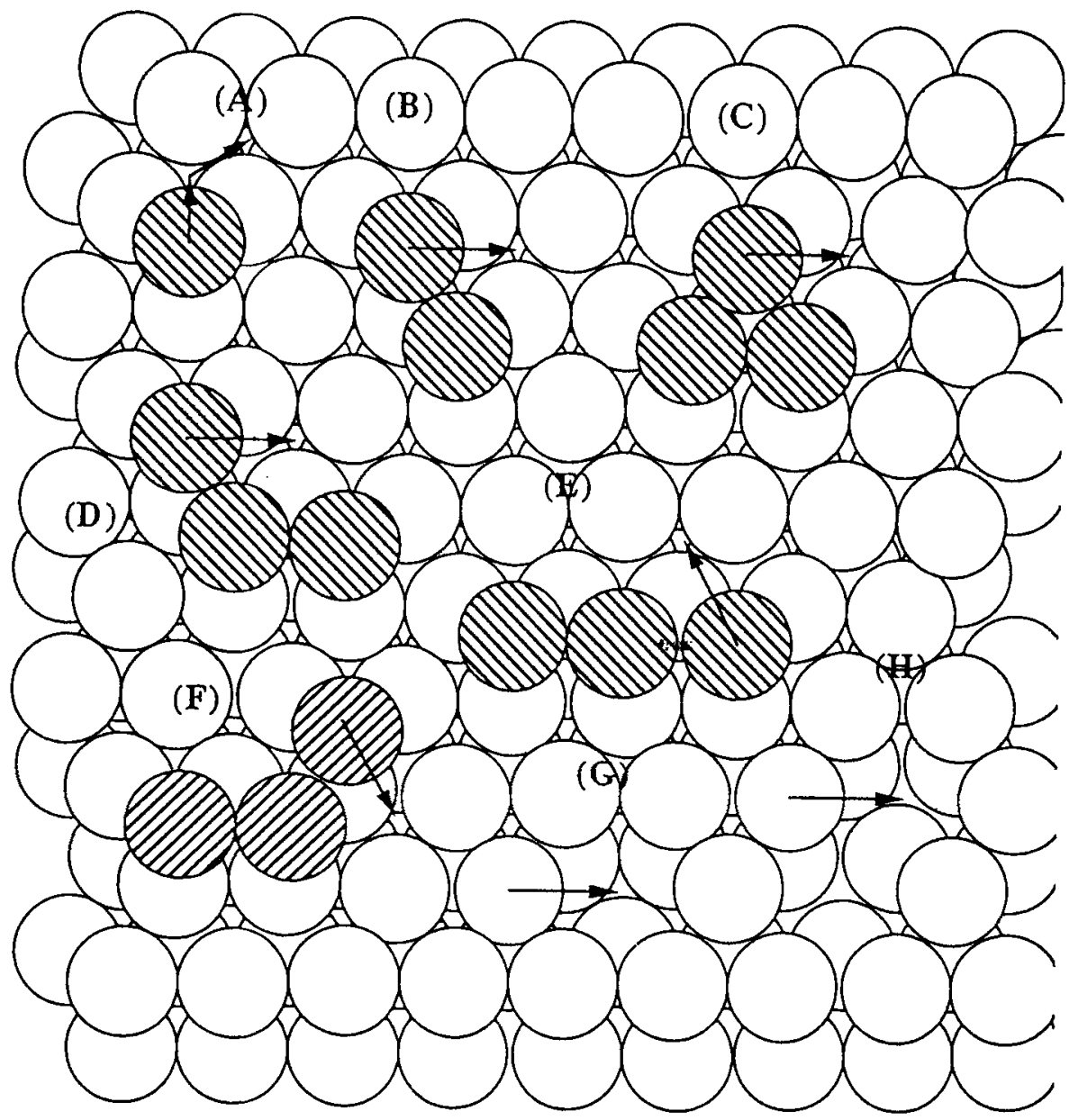

Figure 1. Configuration of adatom and adatom clusters on (111) plane of an fcc metal. A. a single adatom, $\mathbf{B}$. motion of a di-adatom, $\mathbf{C}$. one of dissociation process of tri-adatom, $E_{3}^{60} \rightarrow E_{3}^{120}, \mathbf{D}$. conversion of a tri-adatom, $E_{3}^{120} \rightarrow E_{3}^{60}$, E. $E_{3}^{180} \rightarrow E_{3}^{120}$, F. $E_{3}^{120} \rightarrow E_{3}^{180}$, G. motion of a vacancy on (111), $\mathbf{H}$. motion of a divacancy on (111). 
(i) A single adatom on (111) plane is very easy to move. The activation energies of the motion of a single adatom in copper and silver from an fcc site to a hcp site are $0.055 \mathrm{eV}$ and $0.058 \mathrm{eV}$, respectively. Here an fcc site means that an adatom forms an fcc structure and an hcp site means an adatom forms an hcp structure. The activation energies of the motion of a single adatom in copper and silver from an hcp site to an fcc site are $0.042 \mathrm{eV}$ and $0.054 \mathrm{eV}$, respectively. The difference is due to the elemental stacking fault.
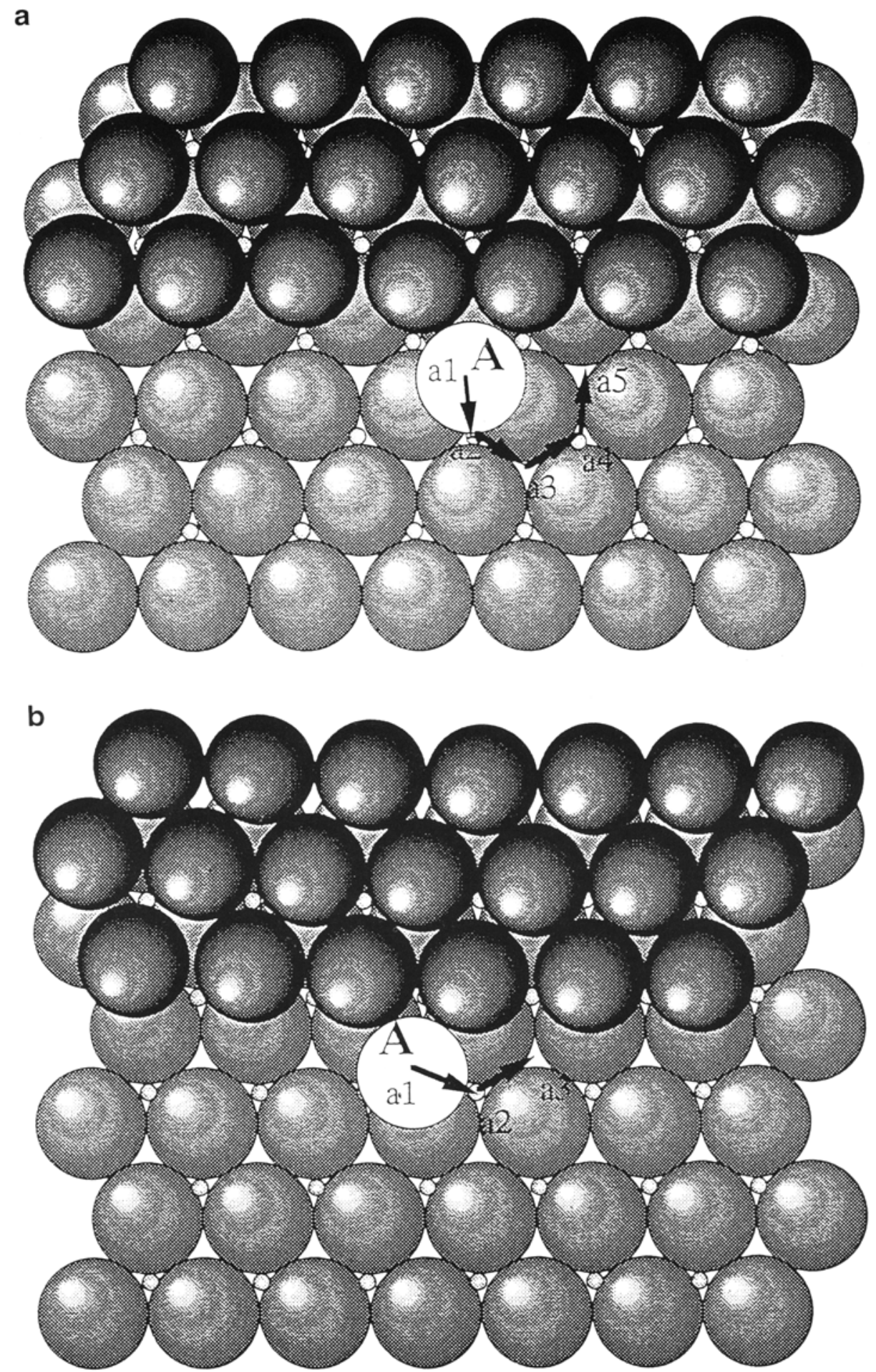

Figure 2. Motion of a trapped adatom to a step on (111). a. Atom A can move $a 1 \rightarrow a 2 \rightarrow a 3 \rightarrow a 4 \rightarrow a 5, \mathbf{b}$. atom A can move through $a 1 \rightarrow a 2 \rightarrow a 3$. 
(ii) The activation energy of the motion of a di-adatom was found to be $0.12 \mathrm{eV}$ in copper, which is much higher than that of a single adatom. A di-adatom is much more difficult to migrate on (111) plane.

(iii) The activation energies of conversion of tri-adatoms in silver are $E_{3}^{60} \rightarrow E_{3}^{120}, E_{3}^{120} \rightarrow E_{3}^{60}, E_{3}^{180} \rightarrow E_{3}^{120}$ and $E_{3}^{120} \rightarrow E_{3}^{180}$ and $0.41,0.08,0.16,0.17 \mathrm{eV}$, respectively. The potential used was as described by Oh and Johnson (1988). The conversion is not very easy.

(iv) A vacancy on (111) is much harder to move.

(v) A single adatom is very easy to move on (111).

\section{Growth of a step on (111)}

Figures $2 \mathrm{a}$ and $\mathrm{b}$ show atom $\mathrm{A}$ sticks to $\mathrm{a}$ flat step. Figure $2 b$ shows atom A sticks to a jog of a step. To move the atom $A$, it has to partially be dissociated from the flat step. For example, the movement of atom A from $a 1$ to a5 proceeds from $a 1 \rightarrow a 2 \rightarrow a 3 \rightarrow a 4 \rightarrow a 5$. However, the motion of atom $A$ shown in figure $2 b$ is different in that the atom A can move without dissociating from the step. Figure 3 shows the crystal energy vs position for copper and silver for the case of figure $2 \mathrm{a}$. This indicates that when an adatom diffuses near the step on (111), the adatom is attracted by the step and it is pulled by the step without an activation energy. In the case of figure $2 a$, a trapped adatom at the step cannot move easily.

When a step on (111) has a jog (figure 4), a trapped adatom $\mathrm{A}$ is rather difficult to move along the step as shown in figure $4 \mathrm{a}$ from $a 1 \rightarrow a 2 \rightarrow a 3 \rightarrow a 4 \rightarrow a 5$, or as shown in figure $4 \mathrm{~b}$ from $a 1 \rightarrow a 2$. It is also more difficult to climb up the jog on (111) as shown in figure 4a, i.e. from $a \mathrm{l} \rightarrow a 2 \rightarrow a 6 \rightarrow a 7 \rightarrow a 8$ or as shown in figure $4 \mathrm{~b}$ from $a 1 \rightarrow a 2 \rightarrow a 4 \rightarrow a 5 \rightarrow a 6 \rightarrow a 7 \rightarrow a 8$. Figures $4 \mathrm{a}$ and

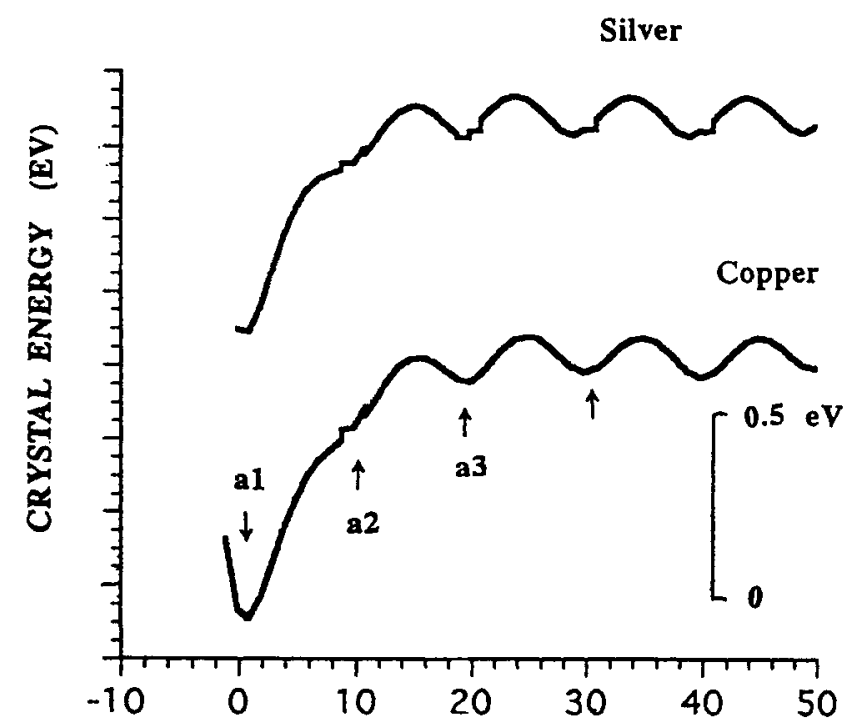

Figure 3. Energy diagram of the motion of a single adatom near a step on (111) for silver and copper. b show the motion of an adatom. The jog has more attractive force to atom A compared with the case without jog.

\section{Monte Carlo simulation of epitaxial growth}

We took a closed packed plane of (111) in an fcc metal. We assumed that atoms of a metal arrive from vacuum at a constant flowing rate. Let an atom's arriving rate at the (111) surface be $\tau_{2}$. The atom adsorbed on (111) can easily diffuse with a low activation energy. Let the mean jumping time from a site to one of the nearest neighbour sites of a single adatom be $\tau_{1}$. $\tau_{1}$ can be expressed as:

$$
\tau_{1}=v_{1} \exp \left(E_{1} /(k T)\right)
$$

where $v_{1}$ is the frequency of the jumping adatom, $E_{1}$ the activation energy of diffusion of a single adatom on (111) plane. $k$ is the Boltzmann constant, and $T$ the absolute temperature. Here we take $\tau_{1}$ as a time unit. Each atom arriving randomly at (111) plane (7d in $x$ direction and $6(3)^{1 / 2} d$ in $y$ direction, where $d$ is the nearest neighbour distance). The periodic boundary conditions are used in $x$ and $y$ directions. Each atom is arriving every $\tau_{2}$ second. In our simulation, two arriving rates are taken, i.e. $\tau_{2}=\tau_{1}$ (case 1); and $\tau_{2}=20 \tau_{1}$ (case 2).

In case 1 , while all the single adatoms on (111) make jumps once a new atom arrives on (111), in case 2, all the single adatoms on (111) make 20 jumps before a new atom arrives on (111). Furthermore, in case 2 there is more time to diffuse before the arrival of another atom. The arrived adatom is put at the fcc site closest to the position given by the random numbers. At every $\tau_{2}$, afl single adatoms are moved to one of the nearest positions by using a random number. As soon as a single adatom forms an adatom cluster, the adatom cluster is frozen and stay at the closest fcc position. All adatoms pass fcc and hcp sites alternately. Figure 5 shows the stacking of atoms on 111 ). While figure 5 a depicts the elapsed time as $600 \tau_{1}=30 \tau_{2}=30$ atoms (slow deposition), figure 5b depicts the elapsed time as $30 \tau_{1}=30 \tau_{2}=30$ atoms (fast deposition) although the deposited atoms are the same as figure 5a. The completeness of the deposition is higher in a slow deposition. Figure $5 \mathrm{c}$ depicts the elapsed time as $110 \tau_{2}=2,200 \tau_{1}=110$ deposited atoms (slow deposition) and figure $5 \mathrm{~d}$ depicts the elapsed time as $110 \tau_{2}=$ $110 \tau_{1}=110$ deposited atoms (fast deposition rate). With a slower deposition rate, the packing density (perfectness) of a deposited film is higher. There is more time to rearrange the crystal for a slower deposition.

\section{Calculation of mean time for an adatom to diffuse on (111)}

The mean jumping time $\tau_{1}$ from a site to one of the nearest neighbour sites of a single adatom is given by 

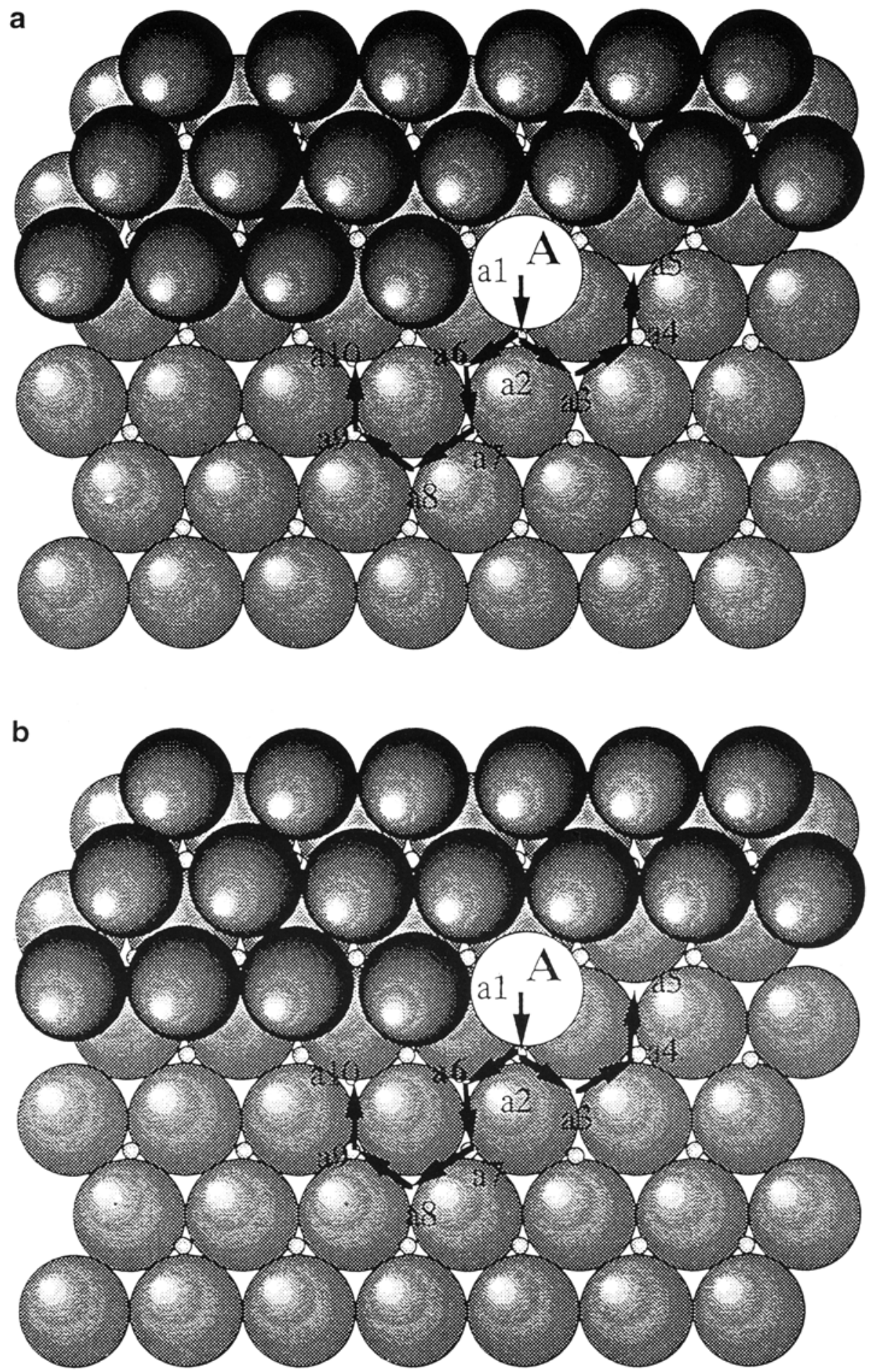

Figure 4. Motion of a trapped adatom to a step with a jog on (111). a. Atom A can move $a 1 \rightarrow a 2 \rightarrow a 3 \rightarrow a 4 \rightarrow a 5$. b. Atom A can move through $a \mathrm{l} \rightarrow a 2 \rightarrow a 3$. To climb a jog is more difficult. Climbing of a trapped adatom to a step on (111). a. Atom A can move $a 1 \rightarrow a 2 \rightarrow a 4 \rightarrow a 5 \rightarrow a 6 \rightarrow a 7 \rightarrow a 8 \rightarrow a 9 \rightarrow a 10$ and $\mathbf{b}$. Atom A can move through $a 1 \rightarrow a 2 \rightarrow a 4 \rightarrow a 5 \rightarrow a 6 \rightarrow a 7 \rightarrow a 8$. 
a

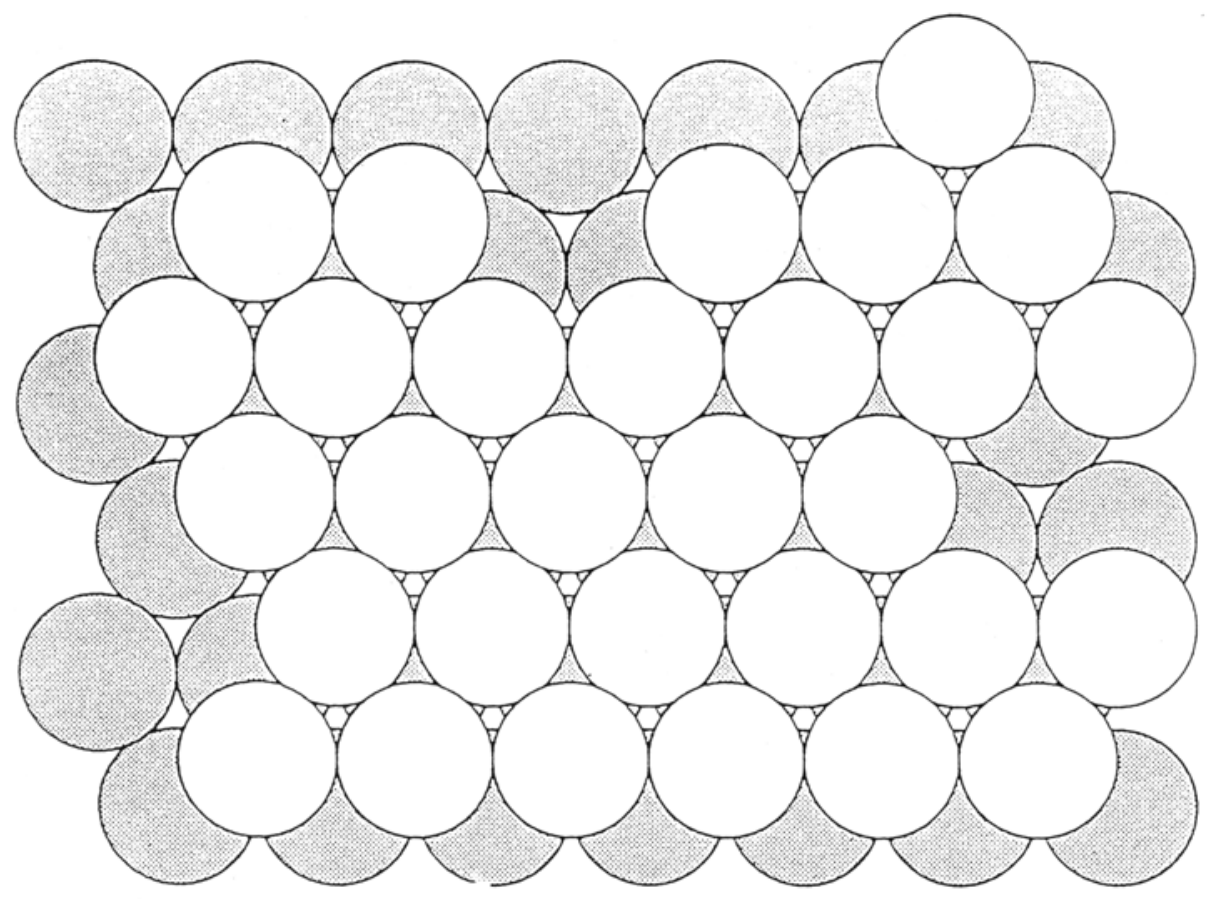

b

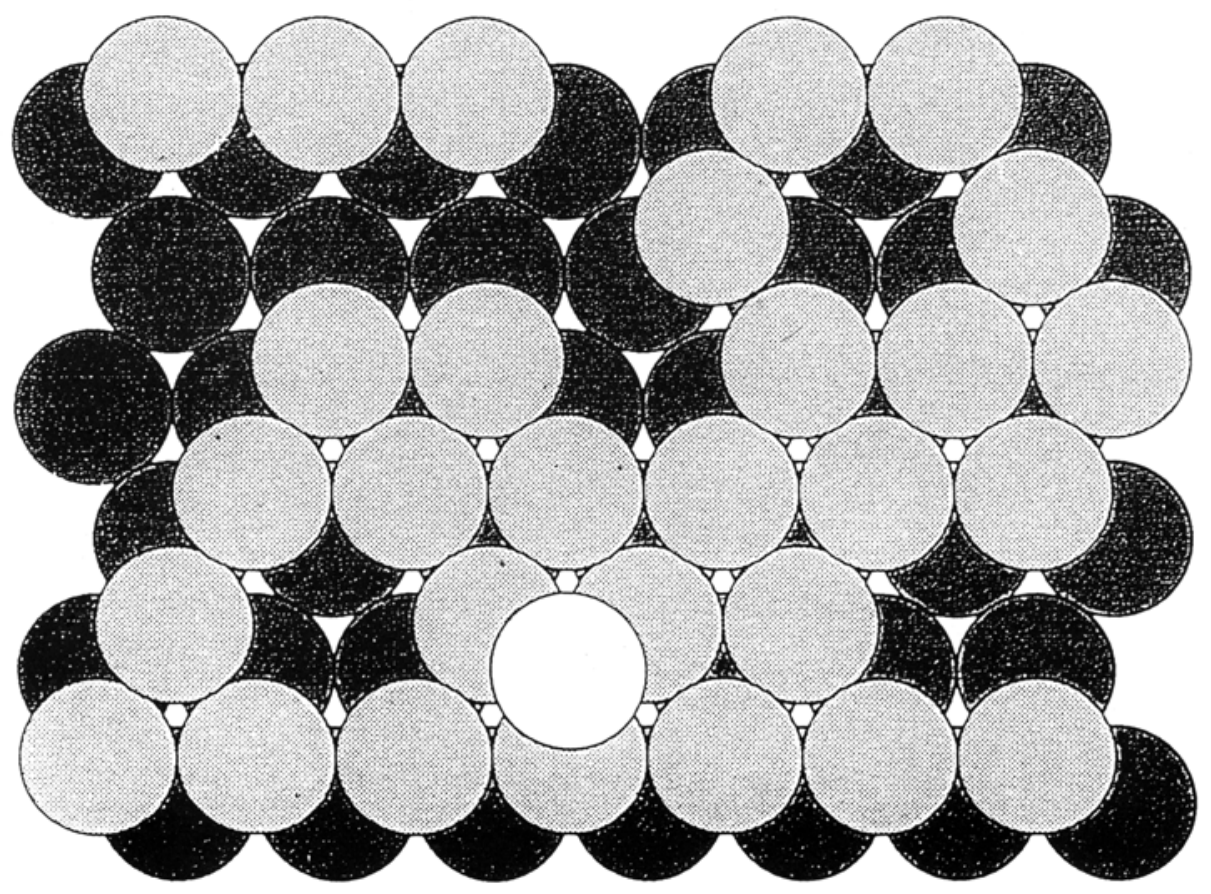

Figure 5. a-b. 
C

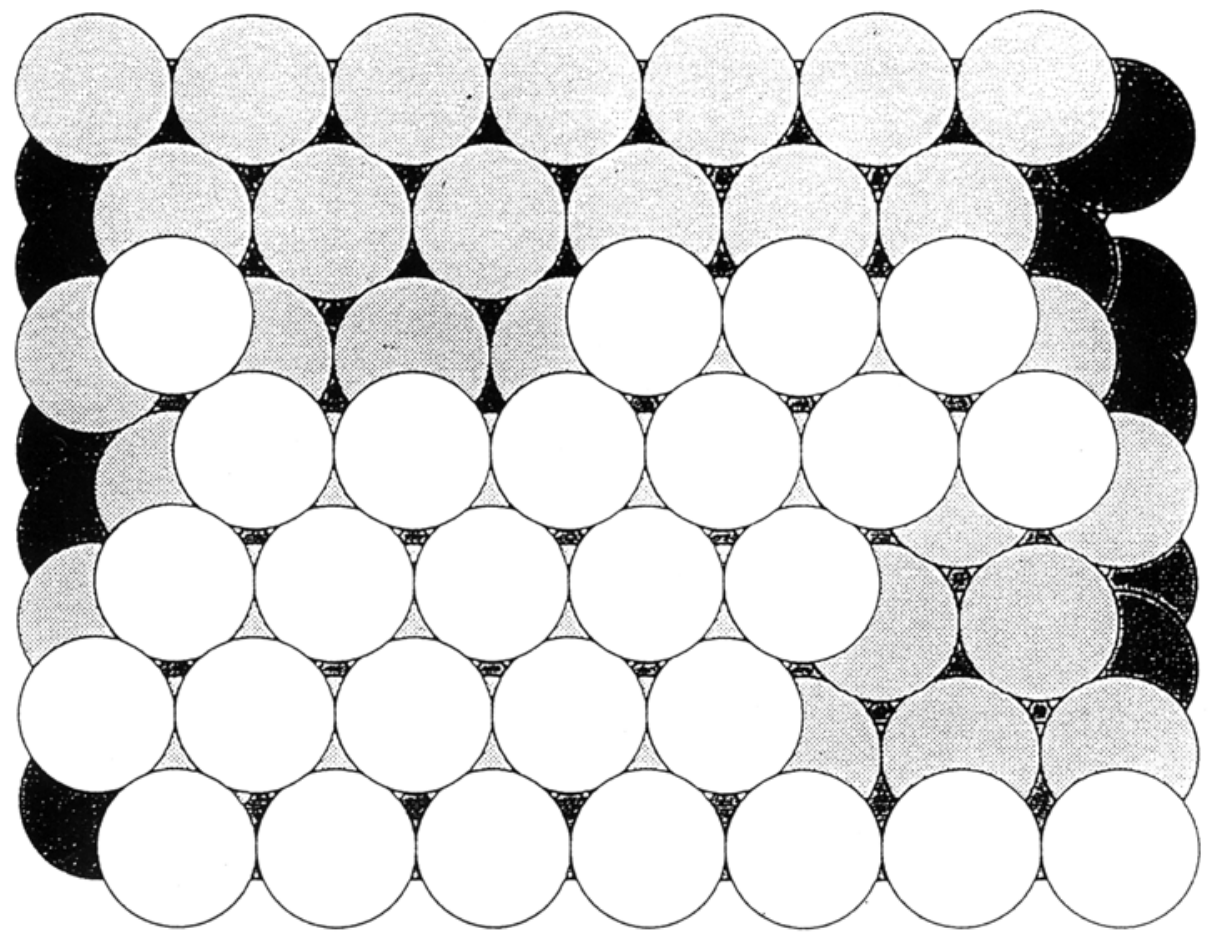

d

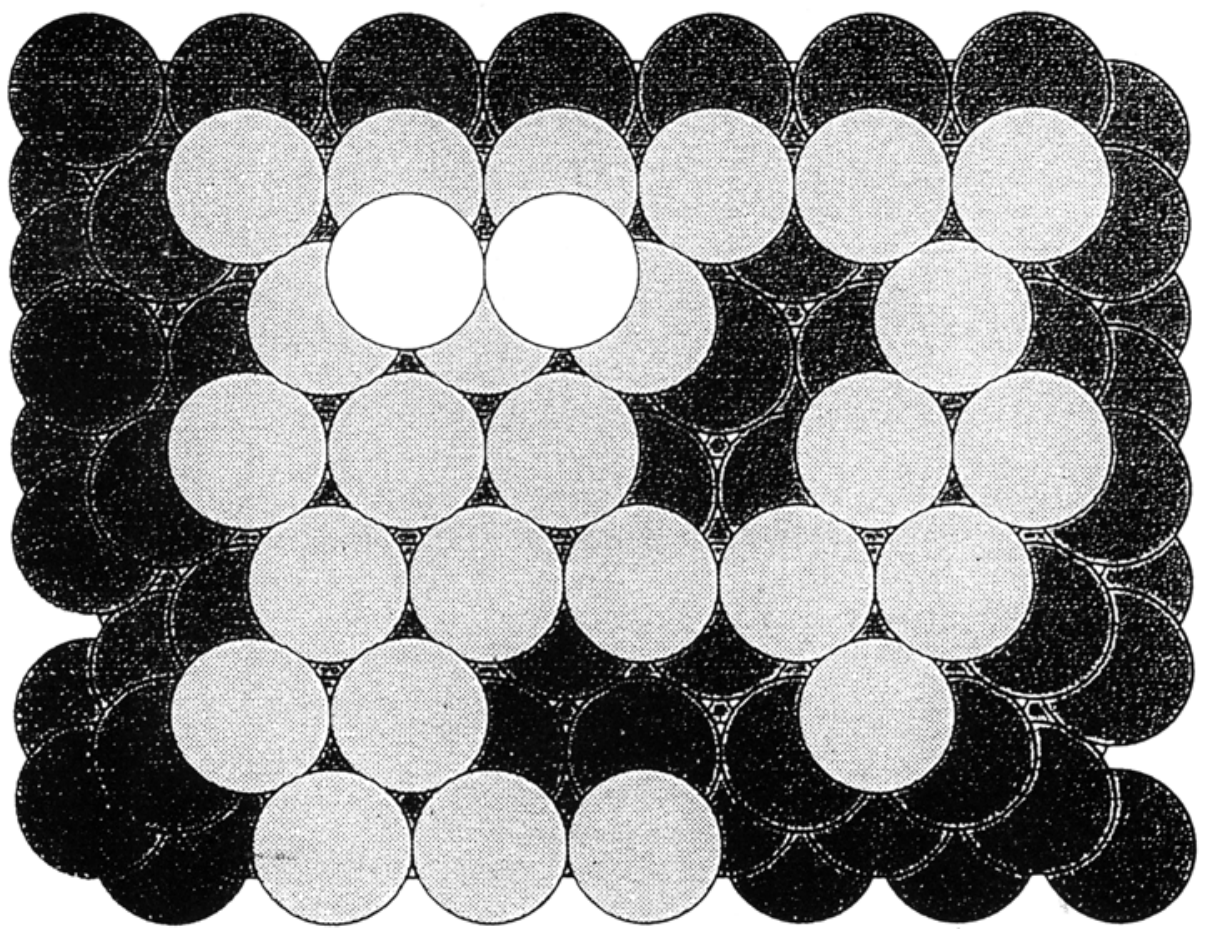

Figure 5. a-d. a. is for the elapsed time $=600 \tau_{1}=30 \tau_{2}=30$ atoms (slow deposition), b. is for the elapsed time $=30 \tau_{1}=30 \tau_{2}=30$ atoms (fast deposition), the deposited atoms are the same as in a. The completeness of the deposition is higher in a slow deposition, c. is for the elapsed time $=110 \tau_{2}=2,200 \tau_{1}=110$ deposited atoms (slow deposition), $\mathbf{d}$. is for the elapsed time $=110 \tau_{2}=110 \tau_{1}=110$ deposited atoms (fast deposition rate). 


$$
\tau_{1}=v_{1} \exp \left(E_{1} /(k T)\right)
$$

where $v_{1}$ is the frequency of the jumping adatom, $E_{1}$ the activation energy of diffusion of a single adatom on (111) plane. $k$ is the Boltzmann constant, and $T$ the absolute temperature. Here we take $\tau_{1}$ as a time unit. $v_{1}$ can be calculated from the energy scheme. $v_{1}$ for copper was of the order of $10^{11} \mathrm{~s}^{-1}$, and $\tau_{1}$ was of the order of $10^{-10} \mathrm{~s}$ at room temperature. At $600 \mathrm{~K}, \tau_{1}$ was of the order of $10^{-11} \mathrm{~s}$.

\section{Conclusions}

We have attempted to combine molecular dynamics and Monte Carlo methods. Molecular dynamics simulates a time sequential phenomena as a function of elapsed time. However, this method is not suited for a problem involving activation processes.

The motion of an adatom along a flat step and with a jog was studied. We observed a relationship between the rate of growth and the packing density of atoms. It was observed to be more suitable for a slow-growth process than for a fast-growth process to obtain a near-perfect single-crystal thin film. The deposition temperatures and the figures based on probability of the crystal growth process have been studied.

\section{Acknowledgements}

The author expresses his thanks to Messrs Yoshinari Abe, Masashi Kato and Tadatoshi Nozaki for their assistance.

\section{References}

Ackland G J, Tichy G, Vitek V and Finnis M W 1987 Philos. Mag. A56 735

Baskes M I 1992 Phys. Rev. B46 2727

Doyama $M$ and Kogure $Y$ To be published

Foiles S M and Daw M I 1986 Phys. Rev. B33 7983

Oh D J and Johnson R A 1988 J. Mater. Sci. 3471

Oh D J and Johnson R A 1989 Atomic simulation of materials (eds) V Vitek and D J Srolovits (New York: Plenum) p. 233

Takano J, Takai O, Kogure Y and Doyama M 1998 Thin Solid Films 31852 